\title{
Reconfigurable Multi-Agent Robots with Mixed Modes of Mobility
}

\author{
Jean-Philippe Clerc \\ Mechanical and Aerospace Engineering \\ University of Florida \\ Gainesville, Fl, USA \\ jpsclerc@ufl.edu
}

\author{
Gloria J. Wiens \\ Mechanical and Aerospace Engineering \\ University of Florida \\ Gainesville, Fl, USA \\ gwiens@ufl.edu
}

\begin{abstract}
This paper focuses on eliminating mobility constraints inherent in current robot locomotion designs, by extending the robot's adaptability and range with mixed/multiple mobility modes. It is assumed that the robots will navigate into a known and controlled environment, potentially equipped with smart structures in order to allow the free movement of the robot. The mechanical designs, kinematic analysis, and the sensor suite of the system are presented including experimental results. An approach to climbing using a modular serial-paired design and a minimal torque-based path planning algorithm is also presented.
\end{abstract}

Index Terms - Modular; Multi-Agent; Serial-paired; Climbing Robot, Urban Inspection.

\section{INTRODUCTION}

The purpose of this research is to introduce a new autonomous multi-agent robotic system designed to be capable of performing urban survey, inspection, surveillance, reconnaissance and/or other similar applications. The research focuses on eliminating mobility constraints inherent in current designs, thus extending the robot's adaptability and range.

Presently the locomotion of most robotic systems can be divided into three main categories: rolling, walking, and reconfigurable approach. While really effective, a rolling robot is restricted to flat horizontal and low grade surfaces. A walking robot is able to climb and access elevated areas, but is often characterized as being slow, having complex motion and balancing issues on flat horizontal surfaces. Reconfigurable robots display a wide range of locomotion and ability to adapt to new tasks by altering their configuration. On the downside, they require many actuators, plenty of power and provide very little room for payloads. Thus there is a need to introduce a robotic system that can be efficient on flat horizontal surfaces in mobility, speed, and simplicity as well as possessing climbing ability.

\section{BACKGROUND}

\section{A. Position System}

The PhaseSpace Inc. position system was used to acquire the robots real time 3D position data. This system consists of four linear CCD (Charge Coupled Device) cameras with 3600 $\mathrm{x} 1$ resolutions at 90 degrees to each other creating a synthetic array of $3,600 \times 3,600$ [1]. The system captures $3 \mathrm{D}$ coordinates of markers at a maximum of 220 frames per second and calculates a sub pixel position up to $30,000 \times 30,000$. The position $(\mathrm{x}, \mathrm{y}, \mathrm{z})$ can be reported with a latency of $20 \mu$-sec over an Ethernet connection. The intent is to use the data in real time control.

\section{B. Smart Structure}

A structure may be defined 'smart' if it possesses integrated devices, allowing the structure to interact with the external environment. A smart structure typically consists of 4 major components: the structure itself, sensors, actuators, and control system. The smart structure used in this paper's experimental tests is illustrated in Fig. 1 with robot prototype 1. It consists of an aluminum frame, four servomotors, limit switches for feedback, eight docking points and a microcontroller.

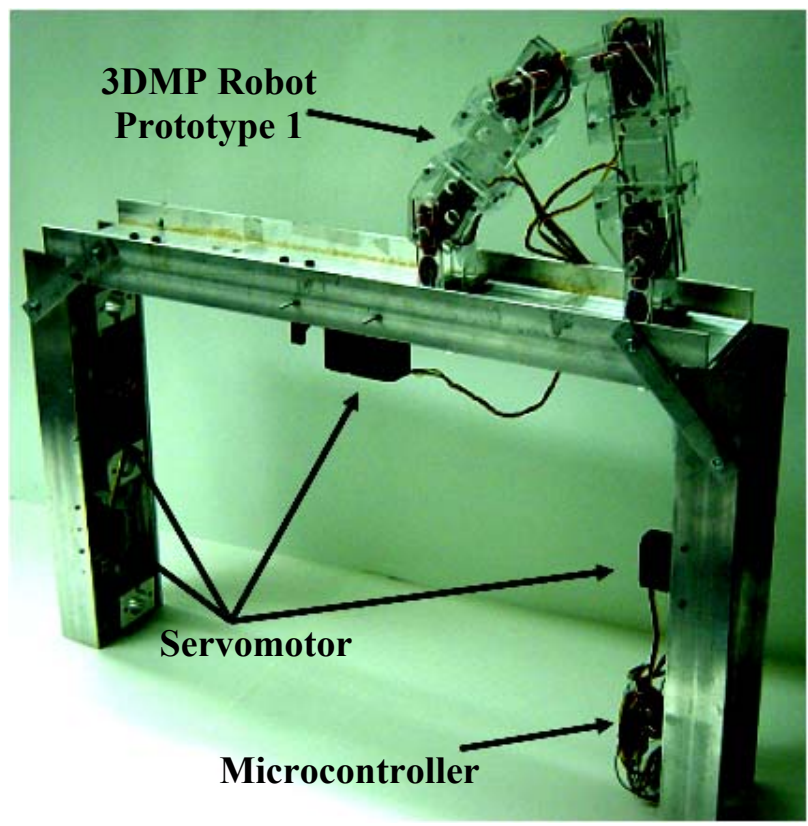

Fig. 1 Prototype 1 Climbing on Smart Structure.

\section{2D TEST PLATFORM}

For the purpose of design validation of the wheeled (rolling) mobility mode, two 2DTP robots were fabricated. The 2DTP acronym stands for Two-Dimension Test Platform, where the two-dimension refers to the mobility restriction of 
these particular robots to flat horizontal surfaces. The 2DTP robots fit within the classification of a Hilare type robot. The algorithms tested on these robots were path planning for inspection and the docking sequence that allows reconfiguration.

\section{A. Design}

A Hilare or differential type robot was designed and custom built in order to validate the design's mobility mode in terms of maneuverability for path execution and docking. Fig. 2 depicts the 2DTP and its major components that are detailed below. The two wheels are located in the front of the robot where each is connected and actuated by a standard servomotor. The robot is battery powered where the battery is located under the robot between the two servomotors. Two IR (infrared) sensors are also placed under the robot and are used for obstacle avoidance. Both the microcontroller and docking mechanism are placed at the rear of the robot. Finally an array of LED's used for position feedback to the controller are located on the top platform of the robot. The arrangement of these components was selected so as to package the robot in terms of balancing of mass distribution and for compactness; while not interfering with functionality.

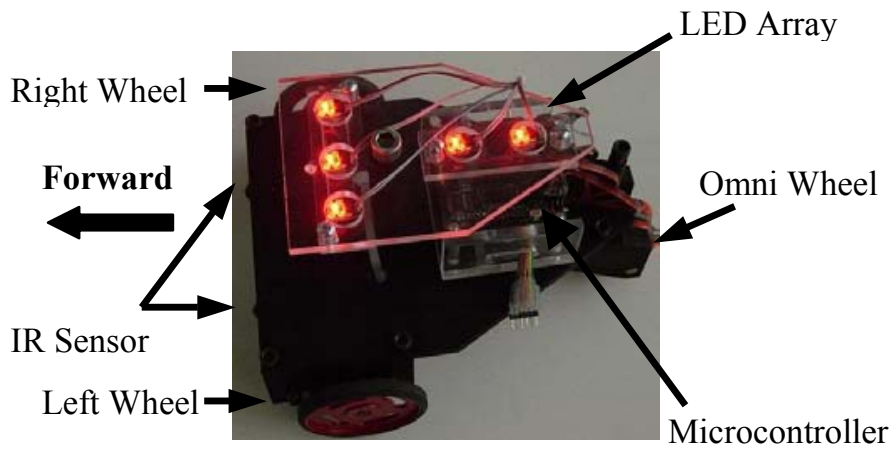

Fig. 2 2DTP With LED's.

\section{B. Docking}

The capability to dock between two agents is critical to the climbing capability of the system. Each robot can move around as single agents and when it is necessary to perform climbing, a docking maneuver is necessary. A simple approach algorithm for docking was created as well as a docking mechanism in order to demonstrate that the robot has the maneuverability necessary to perform docking tasks. Fig. 3 illustrates the docking algorithm sequence: I-II) modify motion to a parallel formation, III) one robot moves to $\mathrm{Y} 1$ position behind the other robot. IV) bottom robot turns $90^{\circ}$ and repositions (V-VI) to align docking mechanism and dock (VII).

\section{Inspection}

In order to further evaluate the above 2DTP design mode, three inspection algorithms were implemented. They were compared in terms of efficiency for five different setups with different obstacles. One of these setups is shown in Fig. 4 consisting of a rectangular area ( $5 \mathrm{ft}$ by $6 \mathrm{ft}$ ) with three obstacles.

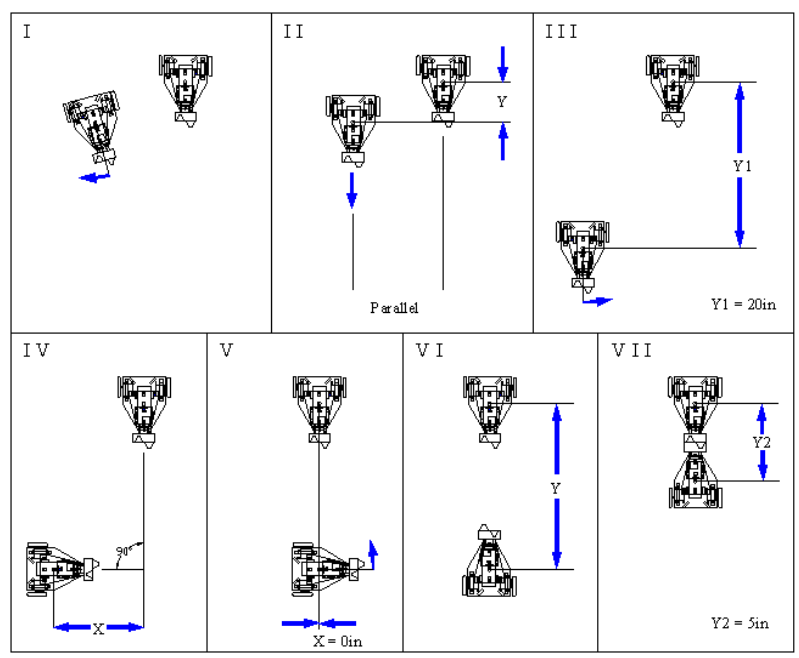

Fig. 3 Docking Operation.

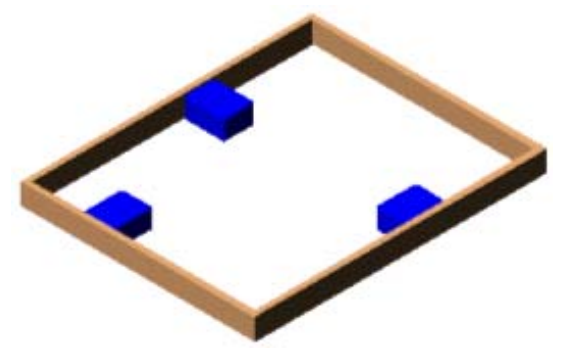

Fig. 4 Test Bed Area.

The resulting percentage of area inspected (coverage) using one robot random, two robots random and teleoperated inspection algorithms are presented in Fig. 5. For all five setups the same trend in results were found, where the teleoperated yields an average of $76.2 \%$ coverage in just 15 minutes of inspection. The average coverage for the two random robot approach was $64.42 \%$ and the one robot approach yields $58.99 \%$ coverage. The low coverage percentage is mainly attributed to the area along the obstacles that could not be inspected due to the IR sensors triggering a change in direction of motion to avoid collisions with the wall and obstacles.

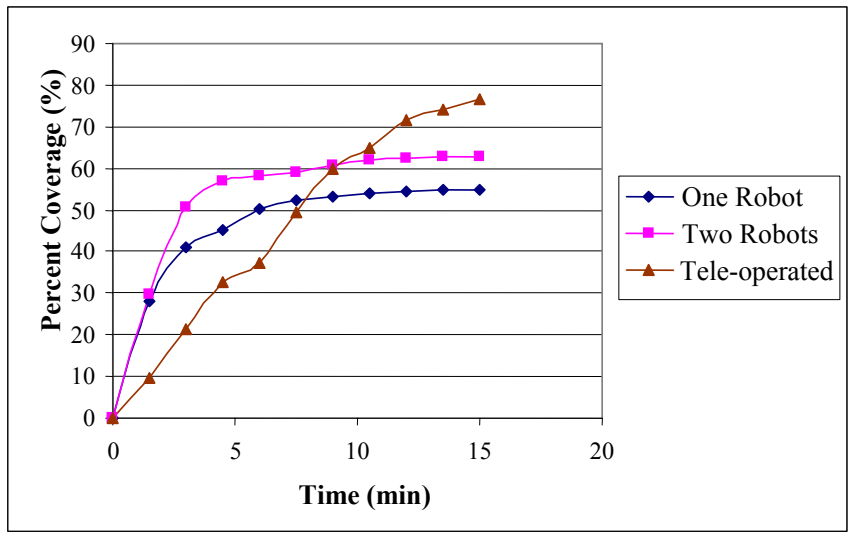

Fig. 5 Coverage Results. 
In order to be able to perform the inspection algorithm described earlier the ability to turn on a 'dime' and to maneuver through tight corners is needed. Fig. 6 illustrates the robot performing wall following around tights corners $\left(45^{\circ}\right)$. The multiple lines are the simultaneous paths of each LED in the array tracked at $30 \mathrm{fps}$ by the position system.

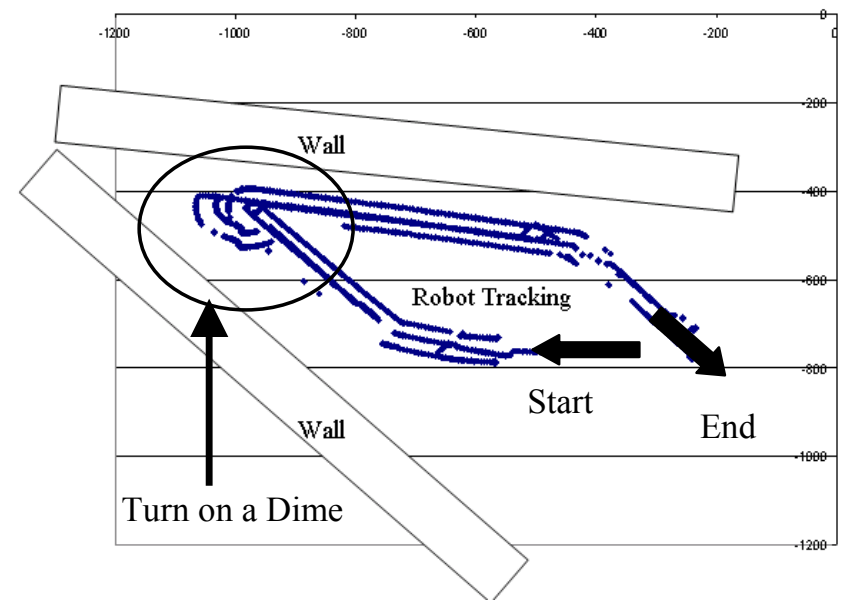

Fig. 6 Mobility Robot Turning $45^{\circ}$.

\section{Climbing}

In order to perform climbing, two identical robots while in their 2DTP mode dock together, back to back, becoming a serial-paired climbing robot. The two robots merged in this configuration are dubbed 3DMP (Three Dimensional Modular Platform) where a modular approach to climbing is investigated. Modular robots generally use a common approach where many similar modules of individually limited mobility (if not zero) are used to provide different types of locomotion as a group (e.g., robots developed at the Xerox Parc [2]). Unlike most modular systems found in the literature, the 3DMP robotic system requires only two modules to perform climbing where each individual module is an autonomous robot. The docking mechanism is designed to allow all robots to be identical and still be able to dock with any other robot. I.e., removing the difficulty of having to find a specific counterpart robot (male/female pair) in order to dock. This section is dedicated to the presentation of the conceptual idea behind the 3DMP, design prototypes, algorithms and results.

\section{A. Design}

The 3DMP robots are individually wheeled autonomous platforms that perform efficiently on horizontal flat surfaces. The two front wheels are actuated using widely available RC servomotors while the third contact point is made using a ball transfer. A ball transfer was integrated on both sides of the robot in order to provide a symmetric robot, no top and bottom. Thus no need to worry about keeping track of what side of the robot is flipped up and what side isn't.

Fig. 7(a) shows two robots docked and posed ready to climb. Fig. 7(b) illustrates one 3DMP robot flipped $180^{\circ}$ above its serial-paired 3DMP robot. In these figures, suction cups illustrate the attachment to climbing surface. Fig. 7(c) depicts the kinematics of the two docked 3DMP robots, and the corresponding constant parameters of the paired 3DMP robots. The constant kinematic parameters for the serial-paired robots are the effective link length parameters $\mathrm{a}_{01}, \mathrm{a}_{12}, \mathrm{a}_{23}, \mathrm{a}_{34}$ and $\mathrm{a}_{45}$ while the variable parameters are $\theta_{1}, \theta_{2}, \theta_{3}$, and $\theta_{4}$ (the four revolute joint angles).
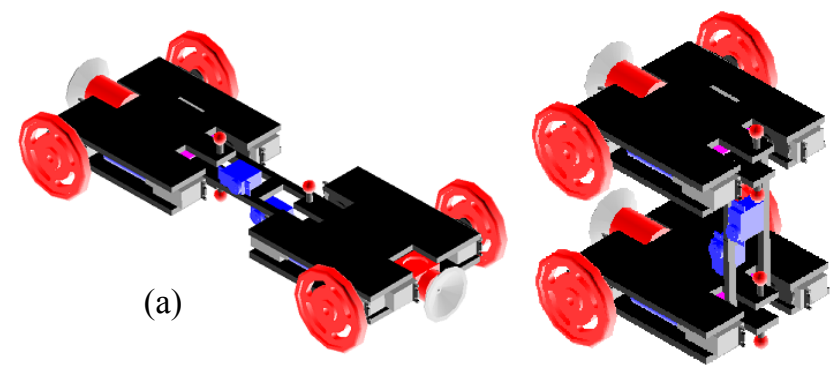

(b)

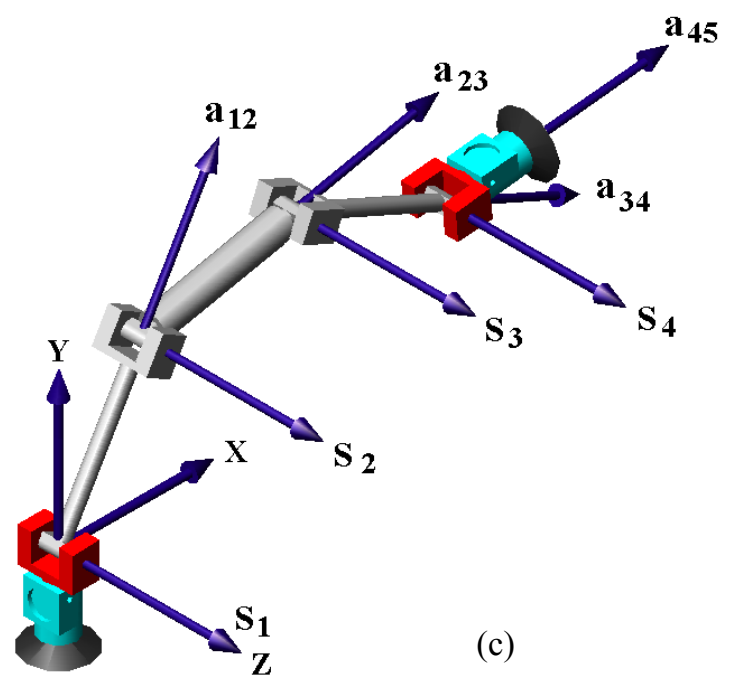

Fig. 7 Kinematic Diagram of 3DMP Robot.

\section{B. Prototypes}

Two prototypes were fabricated in order to perform testing of the merged two 3DMP robots' configuration. The first prototype is two agents permanently joined together in order to simulate the climbing configuration of the system, see Figures 1 and 8 . The second prototype has the capability to roll as an individual robot and to climb as a serial-paired robot, see Fig. 9.

A comparison of the serial-paired robot workspace for each of the two prototypes is presented in Fig. 10, where one end is fixed and the other is free. The range of motion for each joint is the same for both robots $\left( \pm 90^{\circ}\right.$ for all joints). As can be seen in the figure, the given robot design configuration has a void about the joint closest to the fixed (docked) end for prototype 1 . The same general shapes are seen for both prototypes where the actual size and presence of a void is dependent on the kinematic parameter values.

\section{Position Algorithm}

In order to control the serial-paired robot movement, a separate position analysis is required for each set of maneuvers 
for the climbing mode of mobility. To simplify the computation and cut down the computation time, the robot's kinematic model for the climbing maneuver when both ends of the robot are anchored (docked/docking) can be simplified to that of an equivalent four-bar mechanism, see Figures 1 and 11.

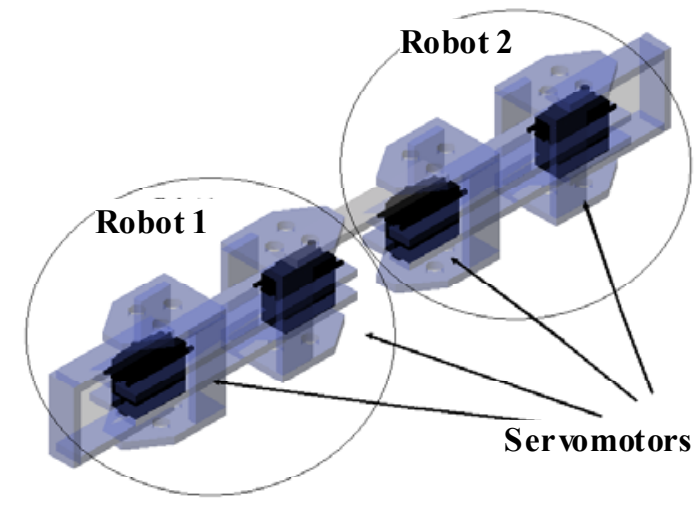

Fig. $8 \quad$ Prototype 1.

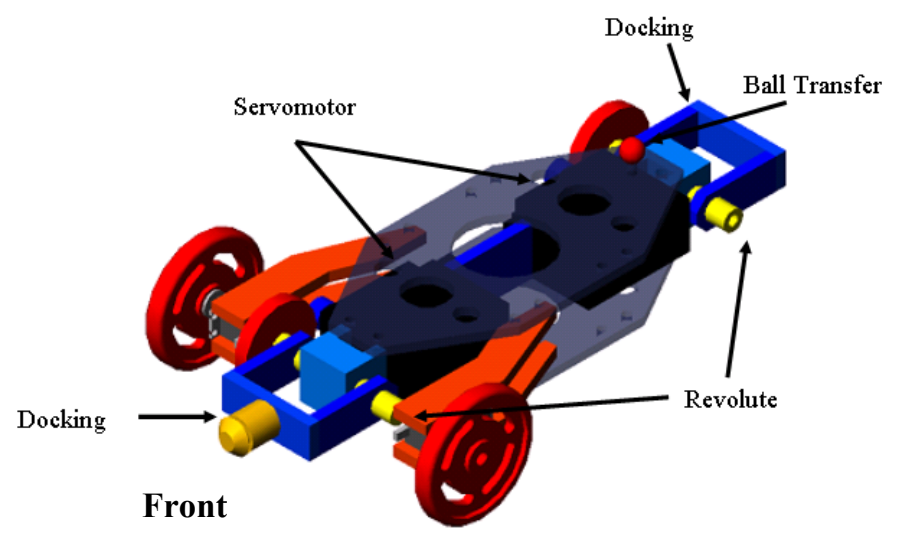

Fig. 9 Prototype 2.

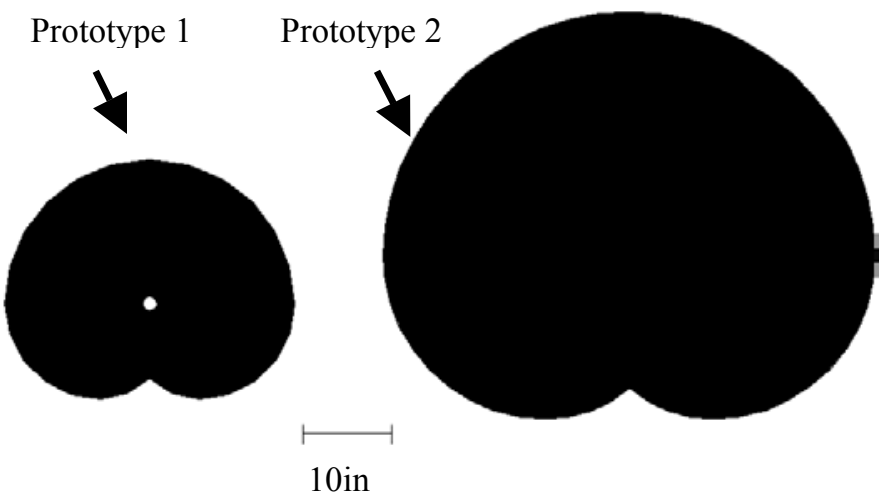

Fig. 10 Workspace of the Two Prototypes.

\section{Torque Algorithm}

Using the Recursive Newton-Euler algorithm [3] for the serial-paired robot model shown in Fig. 12, the required actuator torques of the 3DMP's four joints (T1, T2, T3 and T4) can be determined. In order to simplify the modeling, the following assumptions were made:

- Quasi-dynamic: velocity and acceleration are zero

- The mass of the linkages is modeled as a point mass at their midpoints

- The only external loads present on the mechanism are due to the gravitational forces and actuator torques.

With these assumptions, the set of Newton-Euler equations reduces to the following.

Outward recursive equations:

$$
\begin{aligned}
& { }^{i+1} \dot{v}_{i+1}={ }_{i}^{i+1} R\left({ }^{i} \dot{v}_{i}\right) \\
& { }^{i+1} \dot{v}_{C i+1}={ }^{i+1} \dot{v}_{i+1}={ }^{i+1} \dot{v}_{i} \\
& { }^{i+1} F_{i+1}=m_{i+1}{ }^{i+1} \dot{v}_{C_{i+1}}=m_{i+1}{ }^{i+1} \dot{v}_{i}
\end{aligned}
$$

Inward recursive equations:

$$
\begin{aligned}
& { }^{i} f_{i}={ }_{i+1}^{i} R^{i+1} f_{i+1}+{ }^{i} F_{i} \\
& { }^{i} n_{i}={ }_{i+1}^{i} R^{i+1} n_{i+1}+{ }^{i} P_{C_{i}} \times{ }^{i} F_{i}+{ }^{i} P_{i+1} \times{ }_{i+1}^{i} R^{i+1} f_{i+1} \\
& \tau_{i}={ }^{i} n_{i}^{T}{ }^{i} \hat{Z}_{i}
\end{aligned}
$$

With:

${ }^{i} \dot{v}_{i}=$ linear acceleration of link $i$ expressed in frame $i$

${ }_{i}^{i+1} R=$ rotation matrix defining orientation of frame $i$ relative to frame $i+1$

${ }^{i+1} \dot{v}_{i}=$ linear acceleration of link $i$ expressed in frame $i+1$

${ }^{i+1} \dot{v}_{C i+1}=$ linear acceleration of center of mass of link $i+1$ expressed in frame $i+1$

${ }^{0} \dot{v}_{0}=-g$

$g=$ gravitational force

${ }^{i+1} F_{i+1}=$ inertial force of each link $i+1$ expressed in frame $i+1$

$m_{i+1}=$ mass of link $i+1$

${ }^{i} f_{i}=$ force exerted on link $i$ expressed in frame $i$

${ }^{i} P_{C i}=$ vector which locates the center of mass of link $i$ relative to frame $i$ expressed in frame $i$

${ }^{i} P_{i+1}=$ position of center of joint $i+1$ with respect to center of joint $i$ expressed in frame $i$

${ }^{i} n_{i}=$ torque exerted on link $i$ at joint $i$ expressed in frame $i$

$\tau_{i}=$ torque of jo int $i$

Fig. 13 is a plot of full range of torques possible for each robot configuration as a function of $\theta_{1}$. From this plot, one can extract a piecewise trajectory that will exhibit minimum T1. Fig. 14 shows the resulting torque profiles. It was found that minimal $\mathrm{T} 1$ yielded the best combinations of $\mathrm{T} 1, \mathrm{~T} 2, \mathrm{~T} 3$ and T4 for uniform low values. Hence, the motors for all joints do not require different overall torque capacity. Figures 15 thru 17 illustrate the resulting sequence of configurations for 3 climbing planes of motion: horizontal, $45^{\circ}$, and vertical. 


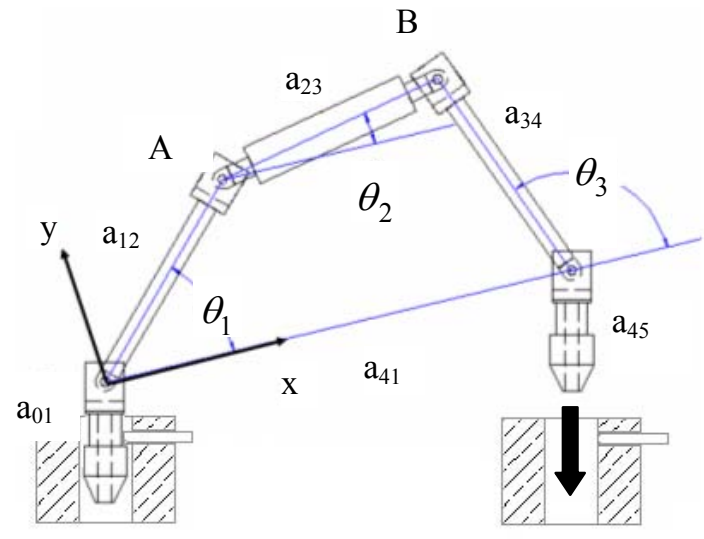

Fig. 11 Instantaneous Four-Bar Mechanism Equivalent.

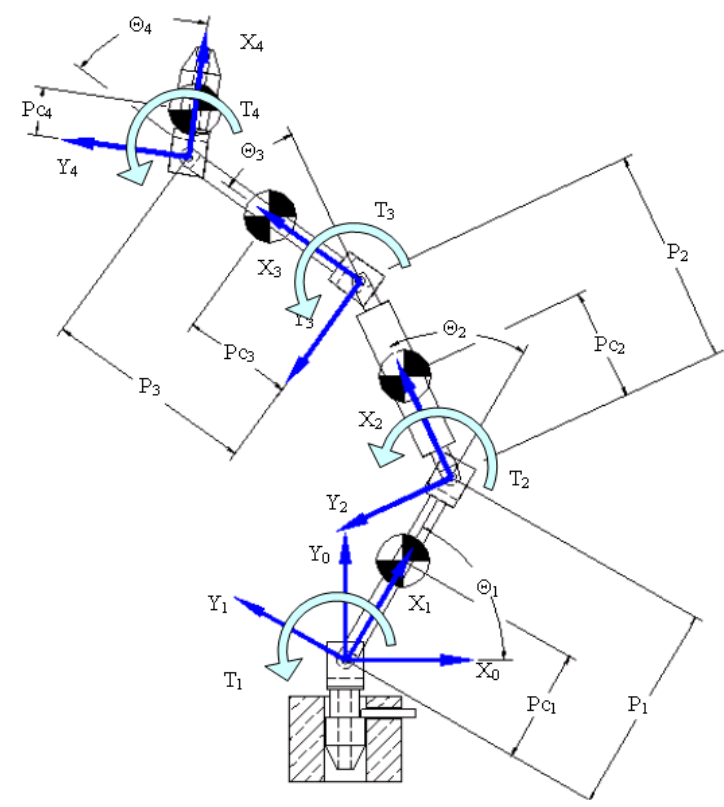

Fig. 12 Diagram for Newton-Euler Equations.

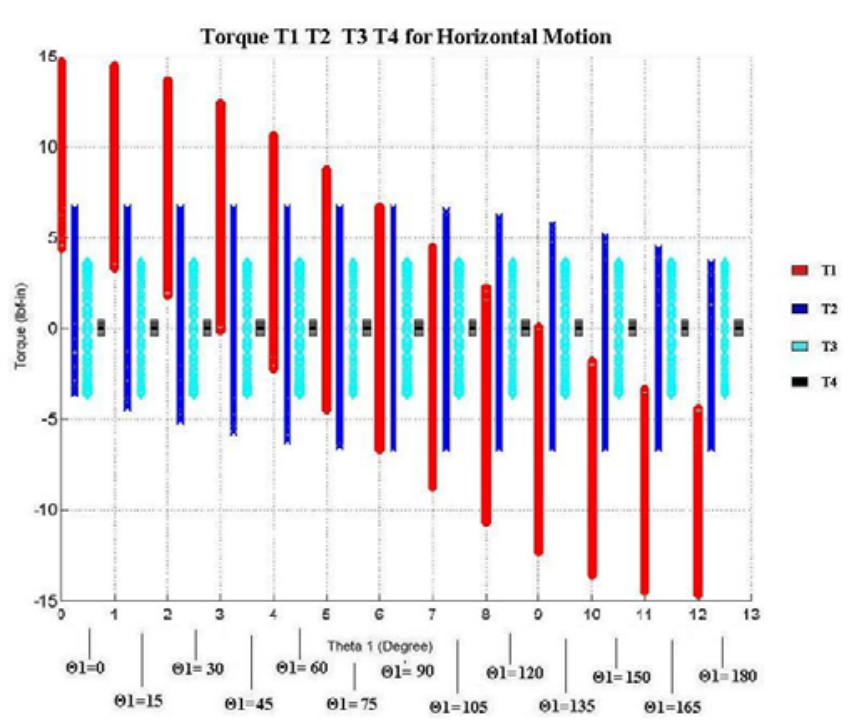

Fig. 13 Torque For Entire Range.

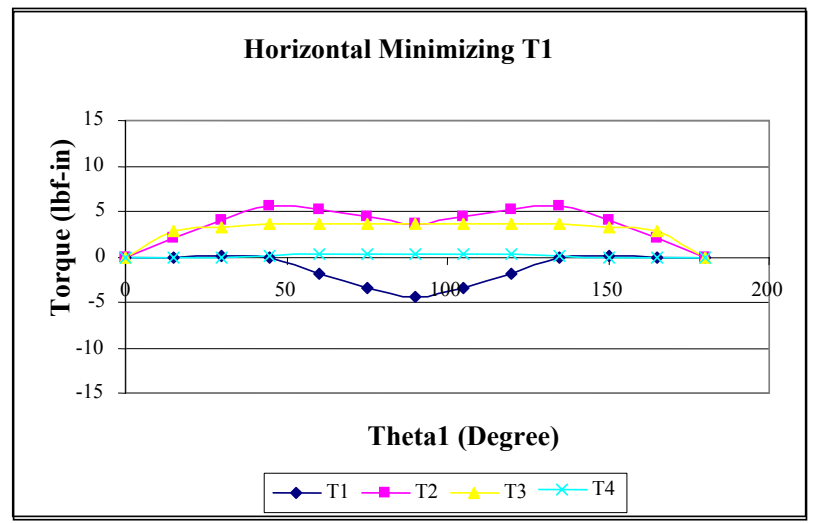

Fig. 14 Torques for T1 Minimized.

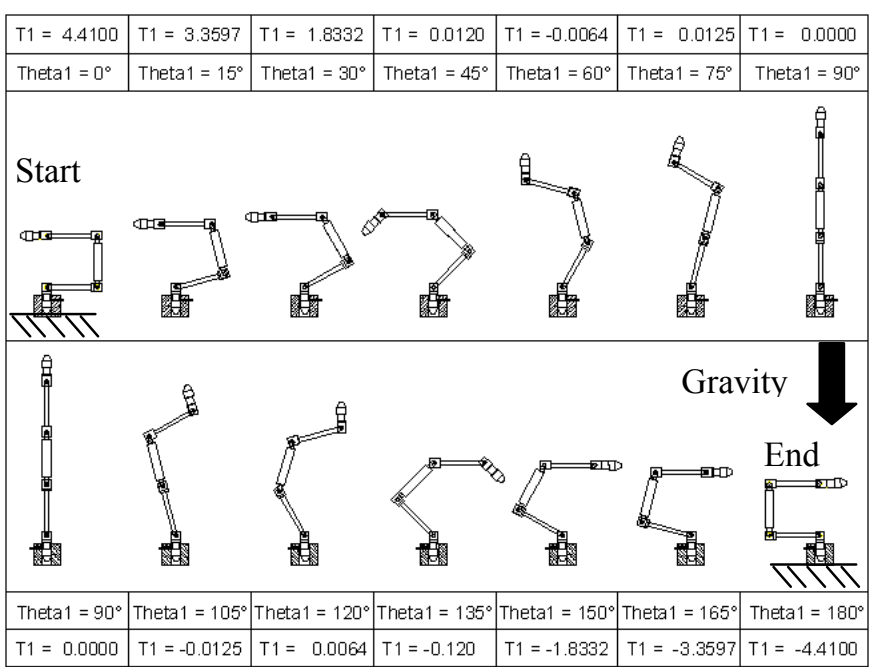

Fig. 15 Lowest Torque While Performing Horizontal Motion.

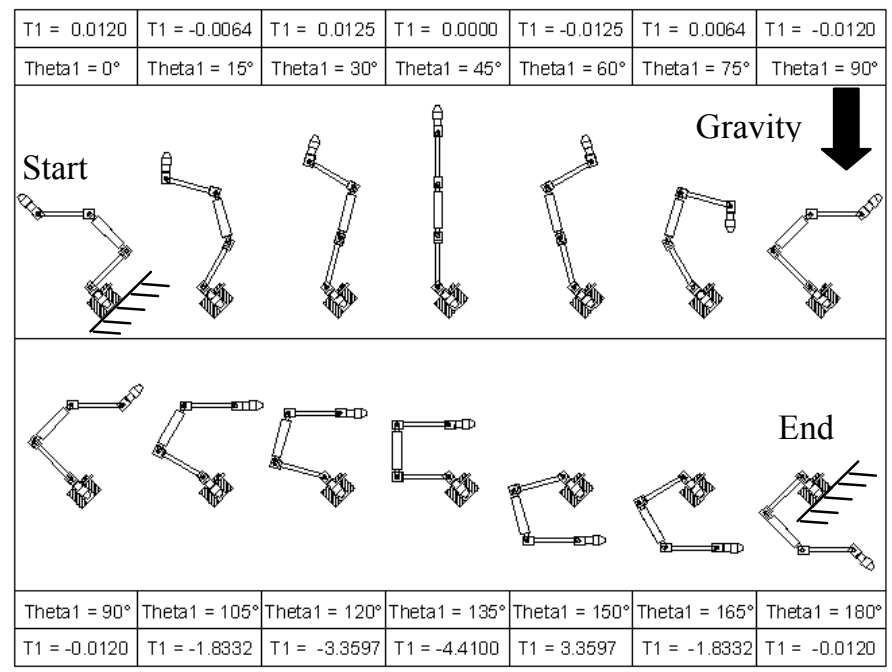

Fig. 16 Lowest Torque While Performing $45^{\circ}$ Motion. 


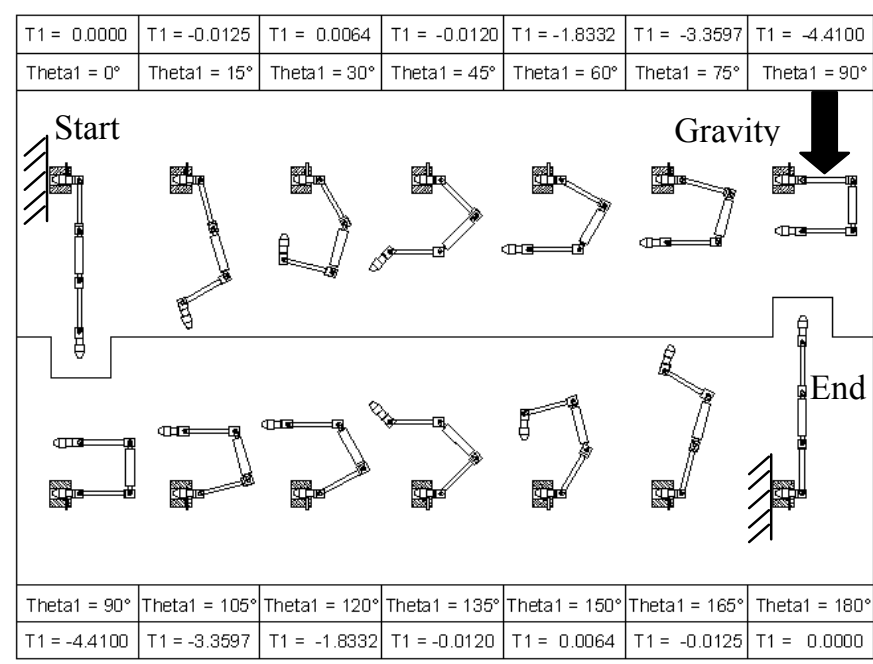

Fig. 17 Lowest Torque While Performing Vertical Motion.

\section{E. Climbing Algorithm}

From the above analysis and observations, the selected climbing mode's path planning methodology is composed of two algorithms: Four-Bar and Torque with the objective of minimizing torque throughout the robot motion for climbing sequences. When the robot is within close range of docking, the Four-Bar algorithm kicks in and computes the angles of the four joints that will allow the docking to occur. The rest of the time the robot is controlled thru the Torque algorithm. A graphical depiction of the climbing is presented in Fig. 18. First stage (I) the Torque algorithm is applied to find the configuration of the robot in the four-bar mode that will yield the minimum torque upon release of one docking port. Then, the four-bar gently undocks from the docking port (II). The Torque algorithm brings the robot close to its next docking port with the lowest possible torque along the path (III). The FourBar algorithm docks the end effector with the structure and the process is repeated again $(\mathrm{V})$. The limitation with this approach is it does not ensure collision free paths between the robot and the structure it is climbing. However, this algorithm provides an effective sequential approach to path planning.

\section{CONCLUSION}

This research presents an innovative robotic systems that can be used for a wide variety of applications. The system is primarily geared towards an autonomous inspection for applications that would require climbing abilities. Several key features have been demonstrated: docking, climbing and inspection. Several inspection algorithms were used to survey a predetermined area with and without obstacles to demonstrate the maneuverability of the design.
The climbing algorithm was developed to accommodate the use of low torque servomotors in the robotic system. A suboptimal path planning solution was achieved by computing the torque at the joints and minimizing the overall torque-based on a minimized T1. Future work will be the refinement of this torque-based path planning algorithm to include collision avoidance of the robot with the structure it is climbing.

\section{ACKNOWLEDGMENT}

The authors would like to acknowledge the contributions of Tracy McSheery and Dmitry Derevyanko from PhaseSpace, Inc. and James Templeman of the Naval Research Laboratory for their assistance with the position system programming and the donation of equipment, respectively.

\section{REFERENCES}

[1] Stan Shepard, Dimitry Derevyanko, October 2002, "Quick Start and Programming Manual”, PhaseSpace Inc.

[2] Mark Yin, et al., February 2002, "Modular Robots", IEEE Spectrum, pp. 30-34.

[3] John Craig, 1989, "Introduction to Robotics Mechanics and Control", Addison Wesley, second edition.-Hankel type involving products of Bessel functions," Phil. Trans. Roy. Soc. London, vol. A247, pp. 529551, April 1955.

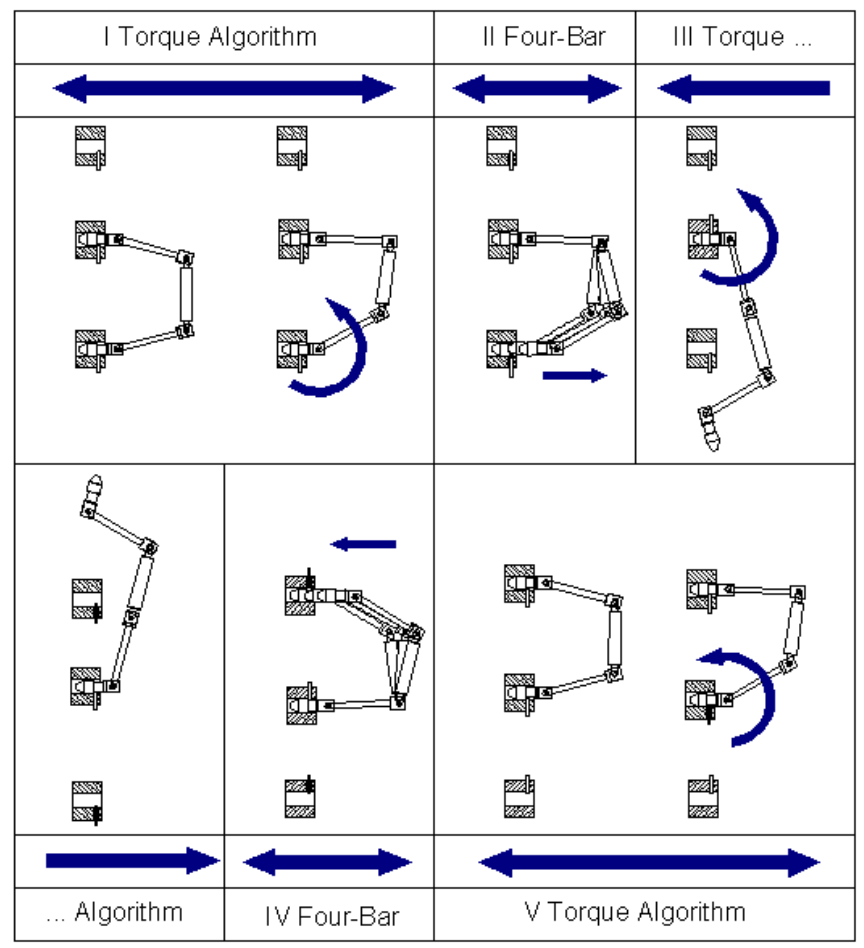

Fig. 18 Climbing Algorithm. 